\title{
Hinokitiol up-regulates miR-494-3p to suppress BMI1 expression and inhibits self-renewal of breast cancer stem/progenitor cells
}

\author{
Shih-Ming Chen ${ }^{1}$, Bing-Yen Wang ${ }^{2,3,4,5}$, Che-Hsin Lee ${ }^{6}$, Hsueh-Te Lee ${ }^{7}$, Jung-Jung \\ Li $^{8}$, Guan-Ci Hong ${ }^{8}$, Yu-Chieh Hung ${ }^{8}$, Peng-Ju Chien ${ }^{8}$, Che-Ying Chang ${ }^{8}$, Li-Sung \\ Hsu $^{1}$ and Wen-Wei Chang ${ }^{8,9}$ \\ ${ }^{1}$ Institute of Biochemistry, Microbiology and Immunology, Chung Shan Medical University, Taichung, Taiwan \\ ${ }^{2}$ Division of Thoracic Surgery, Department of Surgery, Changhua Christian Hospital, Changhua City, Taiwan \\ ${ }^{3}$ School of Medicine, Chung Shan Medical University, Taichung, Taiwan \\ ${ }^{4}$ School of Medicine, College of Medicine, Kaohsiung Medical University, Kaohsiung, Taiwan \\ ${ }^{5}$ Institute of Genomics and Bioinformatics, National Chung Hsing University, Taichung, Taiwan \\ ${ }^{6}$ Department of Biological Sciences, National Sun Yat-sen University, Kaohsiung, Taiwan \\ ${ }^{7}$ Institute of Anatomy and Cell Biology, School of Medicine, National Yang Ming University, Taipei City, Taiwan \\ ${ }^{8}$ Department of Biomedical Sciences, Chung Shan Medical University, Taichung, Taiwan \\ ${ }^{9}$ Department of Medical Research, Chung Shan Medical University Hospital, Taichung, Taiwan \\ Correspondence to: Li-Sung Hsu, email: Ish316@csmu.edu.tw \\ Wen-Wei Chang, email: changww@csmu.edu.tw
}

Keywords: hinokitiol, miR-494-3p, BMIl, breast cancer, cancer stem cells

Received: March 08, $2017 \quad$ Accepted: May 14, $2017 \quad$ Published: June 27, 2017

Copyright: Chen et al. This is an open-access article distributed under the terms of the Creative Commons Attribution License 3.0 (CC BY 3.0), which permits unrestricted use, distribution, and reproduction in any medium, provided the original author and source are credited.

\section{ABSTRACT}

Hinokitiol ( $\beta$-thujaplicin) is a tropolone-related compound that has anti-microbe, anti-inflammation, and anti-tumor effects. Cancer stem/progenitor cells (CSCs) are a subpopulation of cancer cells with tumor initiation, chemoresistant, and metastatic properties and have been considered the important therapeutic target in future cancer therapy. Previous studies reported that hinokitiol exhibits an anti-cancer activity against murine tumor cells through the induction of autophagy. The current research revealed that hinokitiol suppressed the self-renewal capabilities of human breast CSCs (BCSCs) and inhibited the expression of BMI1 at protein level without suppressing its mRNA. Treatment of hinokitiol in mammospheres induced the expression of miR-494-3p and inhibition of miR-494-3p expression in BCSCs. This treatment abolished the suppressive effects of hinokitiol in mammosphere formation and BMI1 expression. BMI1 is a target of miR-494-3p by luciferase-based 3'UTR reporter assay. Overexpression of miR-494-3p in BCSCs caused the down-regulation of BMI1 protein, inhibition of mammosphere forming capability, and suppression of their tumorigenicity. Moreover, miR-494-3p expression was significantly and inversely correlated with patient survival in two independent public database sets. Furthermore, treatment of hinokitiol in vivo suppressed the growth of xenograft human breast tumors as well as the expression of BMI1 and ALDH1A1 in xenograft tumors. In conclusion, these data suggest that hinokitiol targets BCSCs through the miR-494-3p-mediated down-modulation of BMI1 expression.

\section{INTRODUCTION}

Cancer stem/progenitor cells (CSCs) are a subpopulation of cancer cells involved in tumor initiation, resistance to treatment, and metastasis [1-3]. These cancer cells are considered as the most important target in the development of cancer therapy $[4,5]$. In breast cancer, breast CSCs (BCSCs) have been identified as 
cells with surface markers of CD24-CD44+ [6] or high intracellular aldehyde dehydrogenase (ALDH) activity [7]. CSC activity could also be determined by tumorsphere cultivation, a non-adherent culture condition that enables the enrichment of CSC population from cancer cell lines or primary cancer cells [8-10]. The polycomb complex protein BMI1 regulates the self-renewal capability of normal and malignant mammary stem cells [11]. Overexpression of BMI1 in normal mammary epithelial cells increased mammosphere formation [11]. Cooverexpression of Bmil and activated H-Ras (RasG12V) in MCF10A cells resulted in the formation of poorly differentiated carcinomas with epithelial-mesenchymal transition features in severe combined immunodeficient mice [12]. Recently, a small molecule inhibitor of BMI1, PTC-209, has been identified to cause a decrease in BCSCs through the up-regulation of tumor suppressor microRNAs (miRNAs), such as miR-200 and miR-141 [13].

MiRNAs belong to a family of small non-coding RNAs with 19-24 nucleotides in length. This group functions as gene regulators to suppress gene expression by binding to the 3 '-untranslated region to cause translation inhibition or mRNA degradation of their target genes [14]. The functions of miR-494-3p in cancer are controversial. miR-494-3p has been reported to demonstrate oncogenic effects by modulating NOTCH1 and PTEN/PI3K/AKT signaling in non-small cell lung cancer [15], accelerating cell proliferation in liver cancer, and down-regulation or mutation in colorectal cancer [16]. On the other hand, miR-494-3p could inhibit gastrointestinal stromal tumor cell proliferation by targeting KIT [17] or suppressing invasion of prostate cancer cells through down-regulation of CXCR4 [18]. Recently, miR-494-3p was demonstrated to target PAK1 in breast cancer cells, leading to the suppression of cell invasion [19]. However, the role of miR-494-3p in regulation of self-renewal of BCSCs remains unclear.

Hinokitiol is a natural monoterpenoid originally extracted from Taiwanese hinoki and has antiinflammatory and anti-microbial abilities [20, 21]. Furthermore, hinokitiol induces apoptosis in cancer cells through a caspase 3-dependent pathway or through cellcycle arrest [22-24]. Previous studies demonstrated that hinokitiol caused cell death in murine breast and colorectal cancer cells through the induction of autophagy [25]. These studies indicated that hinokitiol could serve as a novel anti-cancer compound. The CSC targeting effect of this compound needs further investigation.

The present study reveals that hinokitiol inhibited the self-renewal of BCSCs and down-regulated BMI1 protein expression without affecting its mRNA level. The expression of miR-494-3p in mammospheres was induced by hinokitiol. Inhibition of miR-494-3p abolished the inhibitory effect of hinokitiol in targeting BMI1. Overexpression of miR-494-3p in human breast cancer cells suppressed tumor growth of BCSCs in vivo. The expression of miR-494-3p was significantly and inversely correlated with breast cancer patient survival in two independent public database sets. Finally, hinokitiol suppressed tumor growth in a xenograft breast cancer model. The upregulation of miR-494-3p as well as downregulation of BMI1 was observed in hinokitiol-treated xenograft tumors. In conclusion, hinokitiol could target BCSCs in vitro and in vivo through upregulation of miR494-3p, inhibiting BMI1 expression.

\section{RESULTS}

\section{Hinokitiol's ability to inhibit the self-renewal capabilities of BCSCs}

The cytotoxic effects of hinokitiol were examined in two human breast cancer cell lines, AS-B145 and BT474. As shown in Figure 1, the half-maximal inhibitory concentration $\left(\mathrm{IC}_{50}\right)$ values of hinokitiol in AS-B145 (Figure 1A) and BT-474 (Figure 1B) cells were 266.9 \pm $42.6 \mu \mathrm{M}$ and $46.5 \pm 8.0 \mu \mathrm{M}$, respectively. The potential anti-CSC effects of hinokitiol was further evaluated in the range of concentrations without causing massive cell death at $0-10 \mu \mathrm{M}$ with a mammosphere cultivation assay. This assay determines the self-renewal capability of BCSCs $[8,26]$. Hinokitiol significantly inhibited primary and secondary mammosphere formation at $10 \mu \mathrm{M}$, a concentration below $\mathrm{IC}_{50}$ value, in both AS-B145 (Figure 1C) and BT-474 (Figure 1D). These results indicate that hinokitiol displays an anti-self-renewal activity of BCSCs in vitro.

\section{The mediation of miR-494-3p on the suppressive effects of hinokitiol to the self-renewal capability and BMI1 expression in BCSCs}

BMI1 positively regulates the self-renewal capability of BCSCs $[11,12]$. The effect of hinokitiol to the BMI1 expression of mammospheres in ASB145 and BT-474 breast cancer cells was examined. As shown in Figure 2, hinokitiol significantly inhibited BMI1 expression in both AS-B145- and BT-474-derived mammospheres at a concentration of $10 \mu \mathrm{M}$ (Figure 2A). BMI1 was further overexpressed in BT-474 cells in order to reduce the therapeutic effects of hinokitiol in targeting BCSCs. The ALDEFLUOR assay findings show that hinokitiol treatment obviously decreased ALDH+ BCSCs within BT-474 mammospheres (from 69.5\% to $20.7 \%$ ), but the inhibition was less efficient in BMI1overexpressing cells (the ALDH+ cells remained 40.6\%) (Figure 2B). However, hinokitiol did not suppress the mRNA expression of BMI1 in AS-B145- or BT-474derived mammospheres (Figure 2C). This result suggests that the inhibitory effects of hinitiol to BMI1 expression may be mediated by a specific miRNA. Previous 
experiments demonstrated that BMI1 was a target of miR-494-3p in oral squamous carcinoma cells [27]. The expression of miR-494-3p in mammospheres was then detected after hinokitiol treatment. Results showed that hinikitiol induced miR-494-3p in mammospheres derived from AS-B145 cells (Figure 3A). When AS-B145 or BT474 cells were transfected with miR-494-3p inhibitor, the inhibitory effects of hinokitiol to BMI1 expression (Figure 3B) or mammosphere formation (Figure 3C) was abolished. The results of the luciferase reporter assay suggest that transfection of miR-494-3p mimic into 293T human embryonic kidney cells or BT-474 breast cancer cells significantly reduced the activity of luciferase fused with wildtype $3^{\prime}$-UTR of BMI1, but not to the luciferase fused with mutated 3'-UTR by deletion of miR-494-3p binding sites (Figure 4A). Furthermore, overexpression of miR-494-3p in AS-B145 and BT-474 mammospheres

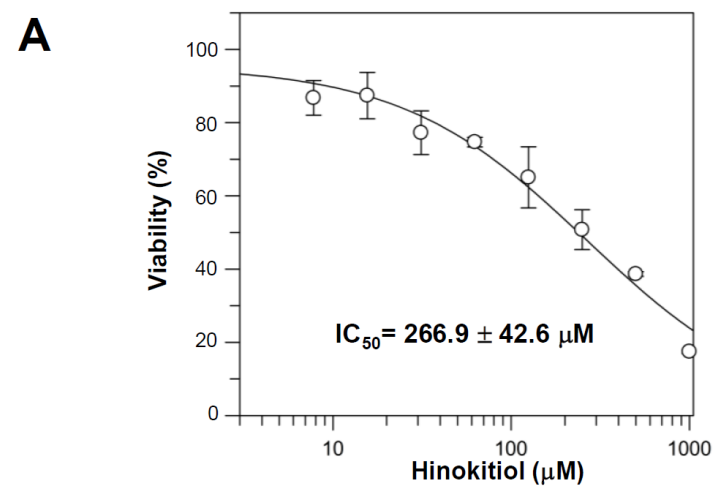

\section{C}

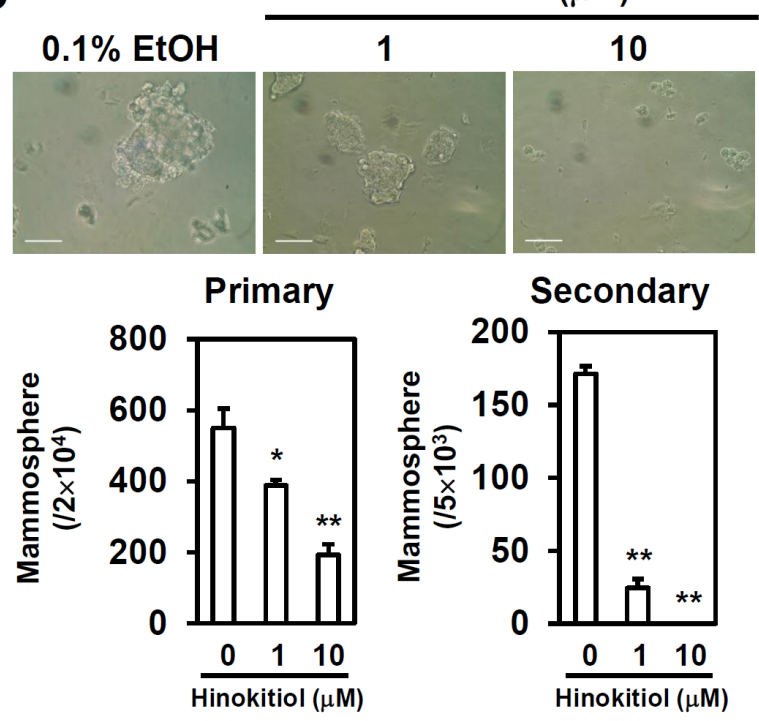

suppressed BMI1 expression (Figure 4B). These results indicate that the inhibitory effect of hinokitiol to the selfrenewal capability of BCSCs is mediated by miR-494-3pinduced BMI1 down-regulation.

\section{miR-494-3p as an oncosuppressor miRNA in breast cancer}

With transfection of miR-494-3p mimic, the overexpression of miR-494-3p significantly suppressed the primary and secondary mammosphere formations of AS-B145 (Figure 5A) and BT-474 cells (Figure 5B). FACS was performed to analyze the intracellular miRNA expression utilizing Smartflare fluorescent beads [28]. In this analysis, BT-474 cells were further sorted into two populations of miR-494-3 $\mathrm{p}^{\text {low }}$ and miR-494-3 $\mathrm{p}^{\text {high }}$ groups with Smartflare miR-494-3p fluorescent probe

B

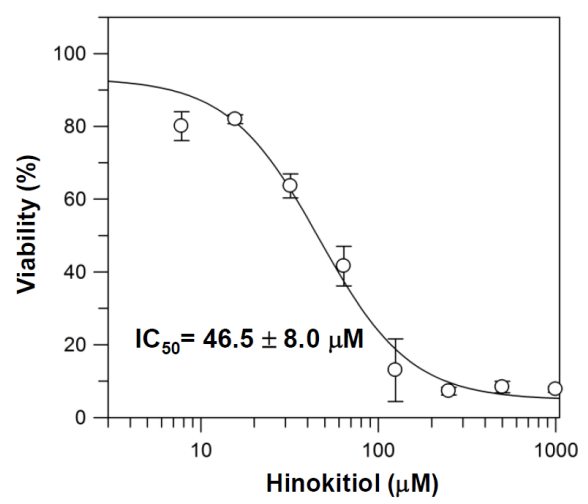

D
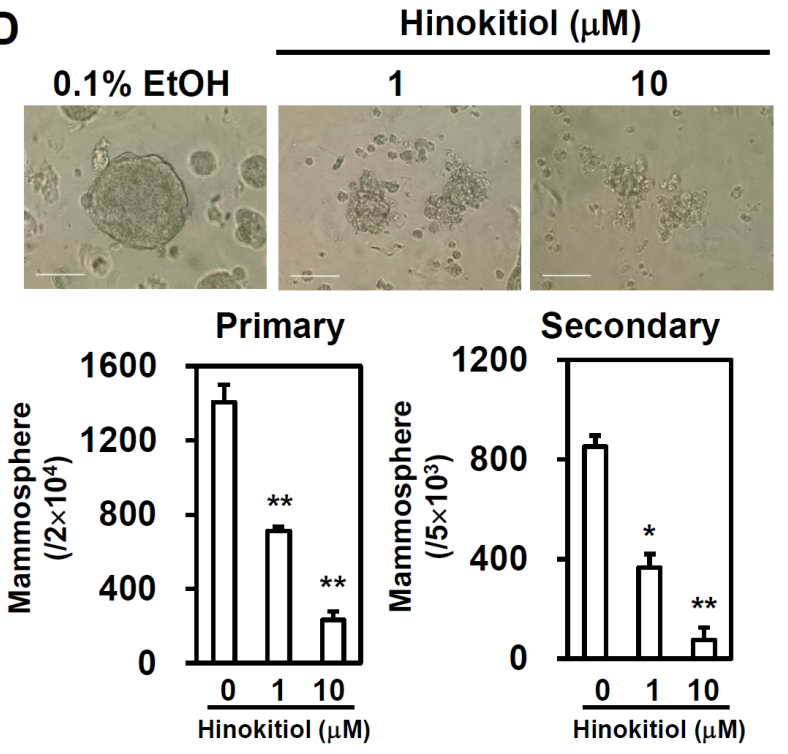

Figure 1: Hinokitiol inhibits self-renewal of BCSCs. (A, B) The (A) AS-B145 and (B) BT-474 human breast cancer cells were treated with various concentrations of hinokitiol for $72 \mathrm{~h}$ and the cell viability was determined by WST1. $\mathrm{IC}_{50}$ values were calculated by GraFit software. (C, D) Self-renewal capability of AS-B145 (C) or BT-474 (D) was determined by mammosphere cultivation. $2 \times 10^{4}$ cells were used for primary mammosphere formation assay and $5 \times 10^{3}$ dissociated primary mammosphere cells were used for secondary mammosphere formation assay. Hinokitiol was treated for 7 days. Scale bar: $100 \mu \mathrm{m} .{ }^{*}, \mathrm{p}<0.05$; ${ }^{* *}, \mathrm{p}<0.01$. The experiments were repeated at least two times and data from one experiment were presented. 
(Figure 5C). Results show that the expression of BMI1 protein was higher in miR-494-3 $\mathrm{p}^{\text {low }}$ BT-474 cells than those of miR-494-3 $\mathrm{p}^{\text {high }}$ counterparts (Figure 5C). With mammosphere assay, the miR-494-3 $\mathrm{p}^{\text {high }}$ BT-474 cells displayed a poor CSC activity that formed less number of mammospheres, as well as decreased in mammosphere size, than the miR-494-3p $\mathrm{p}^{\text {low }}$ counterparts (Figure 5D). The miR-494-3p overexpression was introduced by the lentiviral delivery of miR-494-3p precursor into the BT474 cells and performed xenograftment assay in vivo in NOD/SCID immunocompetent mice; the tumor growth of BT-474 cells with miR-494-3p overexpression was significantly slower than control tumors (Figure 5E, $p=$ 0.0114). Immunohistochemistry analysis demonstrated a reduction of the positive stain of nuclear BMI1 in tumors derived from miR-494-3p overexpressed BT-474 cells
(Figure 5F). Furthermore, the down-regulation of BMI1 protein expression in xenograft tumors derived from miR-494-3p overexpressed BT-474 cells was confirmed by Western blot (Figure 5F). Kaplan-Meier survival analysis was performed in breast cancer patients from GSE37405 dataset (overall survival among ER+ breast cancer patients) by MIRUMIR (Figure 6A) and the Cancer Genome Atlas (TCGA) dataset (metastasis-free survival among invasive breast carcinoma patients) by PROGmiR V2 online tools (Figure 6B). The results showed that the lower expression of miR-494 had a significant poor survival time (Figure 6, $p=0.00113$ for GSE37405 and $p=0.0125$ for TCGA dataset). Moreover, the results indicate that miR-494-3p functions as an oncosuppressor in breast cancer.
A

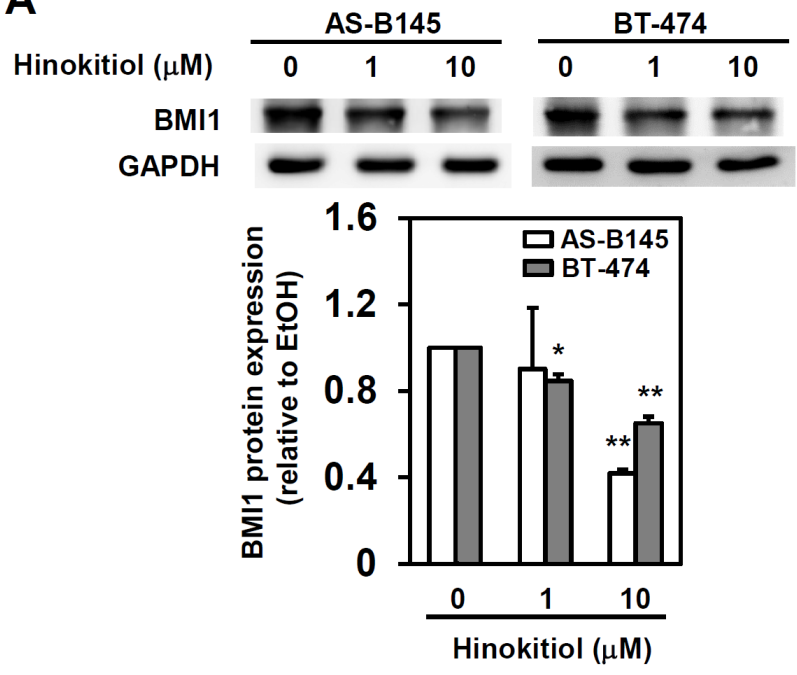

C

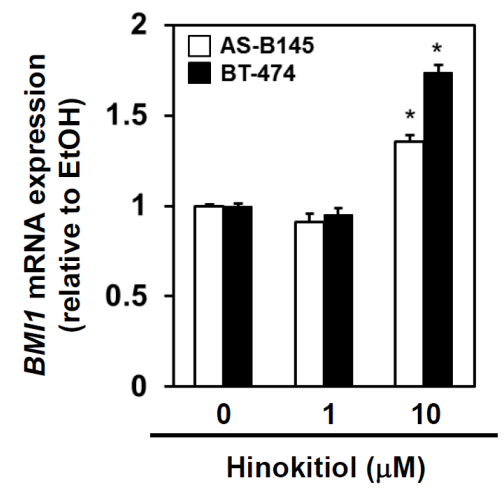

B
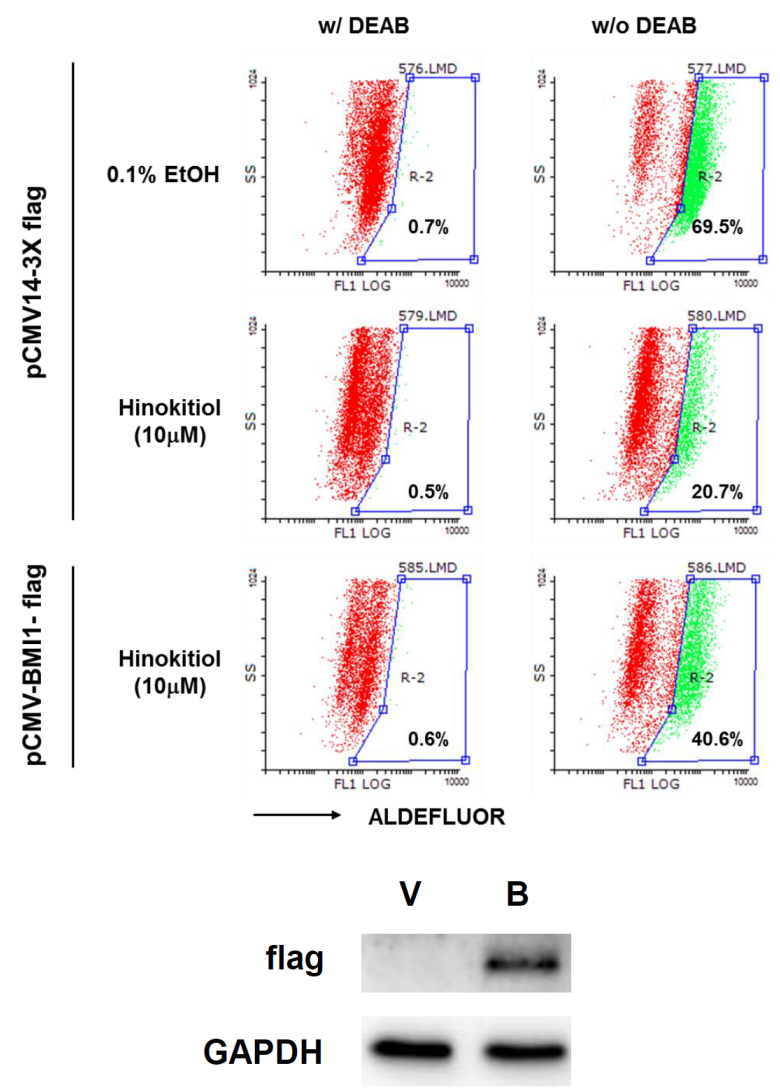

Figure 2: Hinokitiol inhibits BMI1 protein expression, but not mRNA, in BCSCs. (A) AS-B145 or BT-474 cells were cultured into primary mammospheres and dissociated into single cell suspension by HyQTase treatment. Secondary mammosphere cells were then treated with hinokitiol as 1 or $10 \mu \mathrm{M}$ for $48 \mathrm{~h}$ and harvested for analyzing BMI1 protein expression by western blot. BMI1 protein expression levels were normalized to GAPDH and compared with $0.1 \%$ EtOH treated group. ${ }^{*}, P<0.05 ;{ }^{* *}, P<0.01$. (B) BT-474 cells were transfected with pCMV14-3X flag or pCMV-BMI1-flag for 48 hours and performed mammosphere cultivation under $0.1 \%$ ethanol (EtOH) or $10 \mu \mathrm{M}$ hinokitiol treatment. The ALDH+ BCSCs were determined at Day 7 post treatment by ALDEFLUOR assay and FACS analysis. DEAB (N,N-diethylaminobenzaldehyde) was used for gating ALDH+ population of cells. V, pCMV14-3X flag; B, pCMV-BMI1-flag. (C) BMI1 mRNA expression in hinokitiol treated mammopsheres derived from AS-B145 or BT-474 cells was determined by SYBR Green based qRT-PCR. Data were expressed as the mean \pm SD of two independent experiments. 


\section{The anti-breast cancer effect of hinokitiol in vivo associated with the down-regulation of BMI1}

To examine the therapeutic potential of hinokitiol in vivo to breast cancer, the BT-474 mammosphere cells were injected into the mammary fat pads of NOD/SCID and treated with $40 \mathrm{mg} / \mathrm{kg}$ hinokitiol intraperitoneally when the tumor volume reached to $100 \mathrm{~mm}^{3}$. Compared with ethanol-treated group, the tumor growth of hinokitioltreated group was significantly reduced (Figure 7A, $p=0.014)$. By qRT-PCR analysis, the expression levels of miR-494-3p in hinokitiol-treated tumors were significantly increased when compared to ethanol-treated group (Figure $7 \mathrm{~B}, p=0.0002$ ). The BMI1 protein expression level in hinokitiol-treated tumor samples was reduced as compared with the EtOH-treated tumor in Western blot analysis (Figure 7C). By immunohistochemistry analysis, the expression level of ALDH1A1, one of the BCSC markers [7], and nuclear BMI1 was decreased in hinokitioltreated tumors (Figure 7D). These data suggest that the therapeutic effect of hinokitiol in vivo in the suppression of breast tumor growth is associated with the upregulation of miR-494-3p, leading to the down-regulation of BMI1 expression.

\section{DISCUSSION}

Hinokitiol displays an anti-cancer activity by the induction of cell-cycle arrest [23], apoptosis [24], DNA damage [29], or autophagic cell death [25]. This study reports that the anti-CSC activity of hinokitiol was partially mediated by miR-494-3p. The inhibition of miR494-3p expression partially abolished the anti-BCSC effects of hinokitiol (Figure 3). This experiment shows that the anti-cancer activity of hinokitiol is mediated by the induction of an oncosuppressor miRNA. Hibino et al. have demonstrated that inhibition of the enhancer of zeste homolog 2 (EZH2) by 3-deazaneplanocin A induced tumor suppressor miRNAs in liver cancer cells [30]. A similar phenomenon was also found in a recent multiple myeloma study [31]. In hinokitiol-treated xenograft tumors derived from BT-474 mammospheres, the downregulation of EZH2 was observed when compared with EtOH-treated group (Supplementary Figure 1). This finding suggests that hinokitiol may induce miR-494-3p expression through the down-regulation of EZH2, but the underlying molecular mechanism requires further investigation. Previous experiments reveal that hinokitiol could induce proteasomal degradation of epidermal growth
A

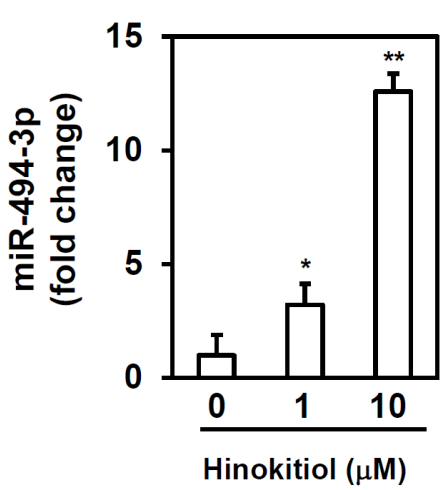

B

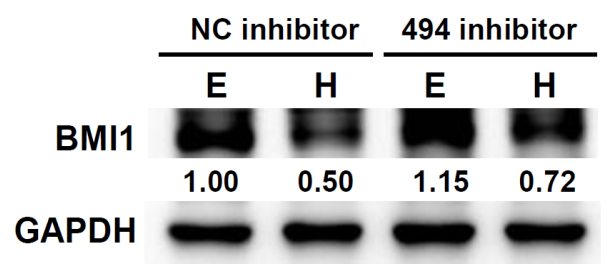

C
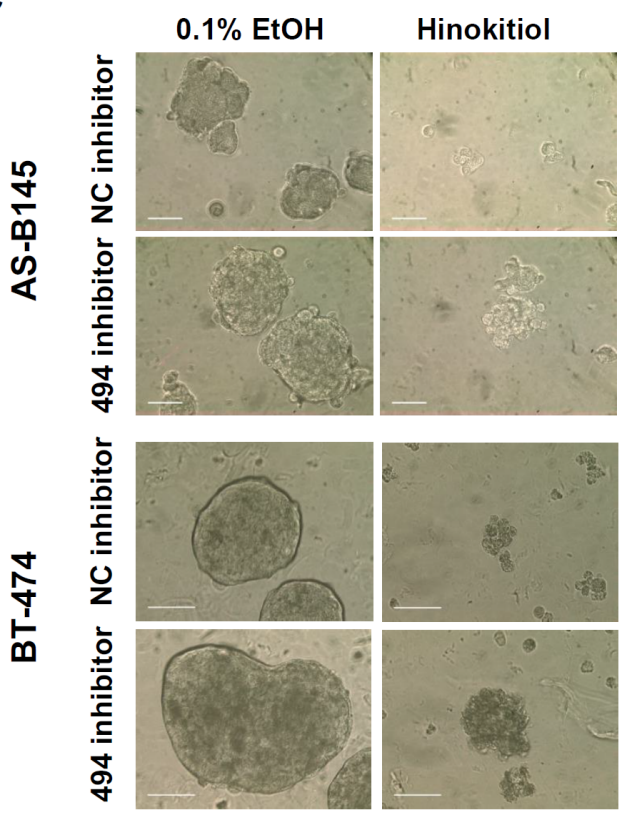

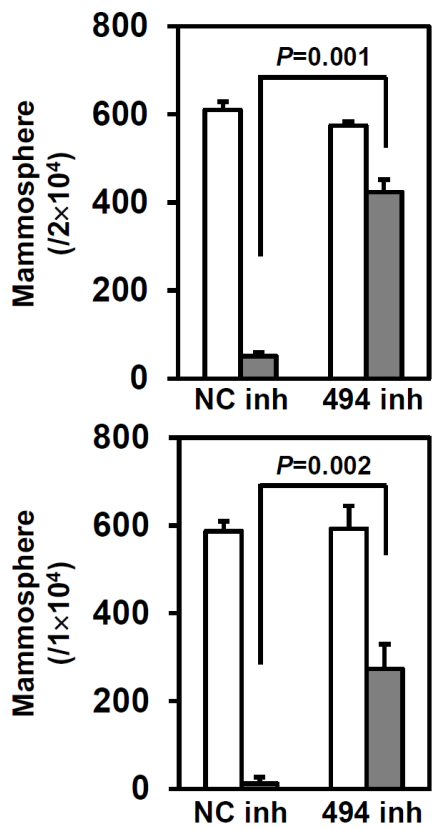

Figure 3: miR-494-3p mediates the suppressive effect of hinokitiol in the self-renewal of BCSCs. (A) miR-494-3p expression in mammospheres derived from AS-B145 cells at Day 6 post hinokitiol treatment were determined by qRT-PCR. ${ }^{*}, P<0.05 ;{ }^{* *}$, $P<0.01$. (B) AS-B145 mammosphere cells were transfected with $100 \mathrm{nM}$ of negative control inhibitor (NC inh) or miR-494-3p inhibitor (494 inh) for 24 hours and treated with $0.1 \% \mathrm{EtOH}$ or $10 \mu \mathrm{M}$ hinokitiol for further 48 hours. Cells were harvested for determination of BMI1 expression by western blot. (C) AS-B145 or BT-474 cells were firstly cultured into primary mammospheres, dissociated into single cell suspension, transfected with $\mathrm{NC}$ inh or 494 inh for 24 hours and performed secondary mammosphere cultivation under the treatment of $0.1 \% \mathrm{EtOH}$ or $10 \mu \mathrm{M}$ hinokitiol. Secondary mammosphere number was counted at Day 7 and data were expressed as the mean $\pm \mathrm{SD}$ of triplicate determinations. White bar, EtOH treated group; gray bar, hinokitiol treated group. Scale bar= $100 \mu \mathrm{m}$. The experiments were repeated at least two times and data from one experiment were presented. 
factor receptor (EGFR), which led to the suppression of vasculogenic mimicry activity of BCSCs [32]. The EGFR signaling has been demonstrated to contribute to the enhancement of BCSC self-renewal induced by versican [33] or by tumor-associated macrophages [34]. Downregulation of EGFR expression by hinokitiol may also involve in the anti-BCSC effect but remains to be further investigated. The relationship between the expression of miR-494-3p and EGFR in breast cancer cells will be further investigated.

The function of miR-494-3p in carcinogenesis is controversial. Several reports suggested that miR-494$3 p$ is an oncogenic miRNA. This miRNA shortens the disease-free survival time in lung cancer patients with high expression levels [15] or the inhibition of PTEN by miR494-3p, leading to the activation of Akt in nasal natural killer cell lymphoma [35]. By contrast, other reports indicated that miR-494-3p could be a tumor suppressor miRNA. The direct targeting of Sox 9 by miR-494-3p led to the suppression of cell migration, invasion, as well as tumor growth of chondrosarcoma cells [36]. The dual role of a single miRNA in carcinogenesis would not be surprising. miR-375 functioned as both an oncomiR and tumor suppressor miRNA in prostate cancer, depending on the stage of tumor progression and hormone status [37]. Furthermore, the effect of miRNAs to carcinogenesis mainly depends on the cellular context in different tumors
[38]. This study presents the tumor-suppressive function of miR-494-3p in breast cancer by inhibiting BCSC selfrenewal and directly targeting BMI1 (Figure 4 and 5). These findings are consistent with the previous studies, which demonstrated that miR-494-3p induced cellular senescence in oral squamous carcinoma cells through the downregulation of BMI1 [27]. These results also show the tumor suppression effects of miR-494-3p. In addition, miR-494-3p was negatively correlated with the overall survival of ER+ breast cancer patients (GSE37405) (Figure 6A) or metastasis-free survival of breast invasive carcinoma patients (TCGA data) (Figure 6B). These data were consistent with a recent study from Zhan et al., showing that the expression of miR-494 was significantly reduced in tumors when compared with adjacent nonneoplastic breast tissues [19]. Altogether, these data strongly suggest that miR-494-3p is an oncosuppressor miRNA in breast cancer.

The current research shows that irradiation could induce epithelial-mesenchymal transition in breast cancer cells by the upregulation of BMI1 [39]. Knockdown of BMI1 abolished irradiation-induced cell migration in breast cancer cells [39]. Results regarding the inhibitory effect of hinokitiol in BMI1 expression through the induction of miR-494-3p suggest that hinokitiol has the potential to develop into a sensitization agent in breast cancer radiotherapy. On the other hand, utilization of

\section{A}
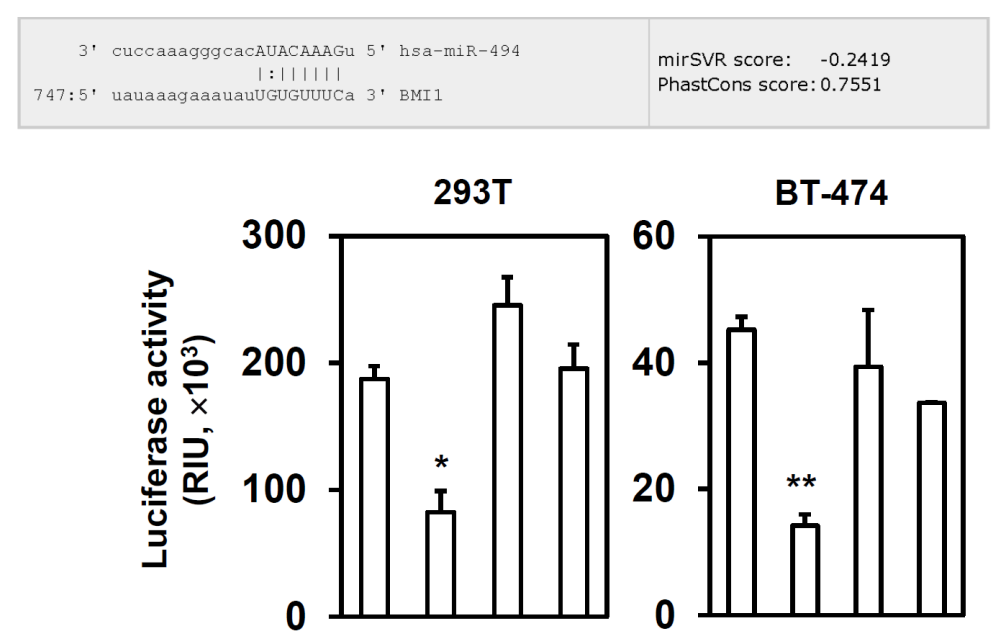

\begin{tabular}{|l|c|c|c|c|}
\hline Bmi1 3'UTR WT & + & + & - & - \\
\hline Bmi1 3'UTR del 762-768 & - & - & + & + \\
\hline NC mimic & + & - & + & - \\
\hline 494-3p mimic & - & + & - & + \\
\hline
\end{tabular}

B

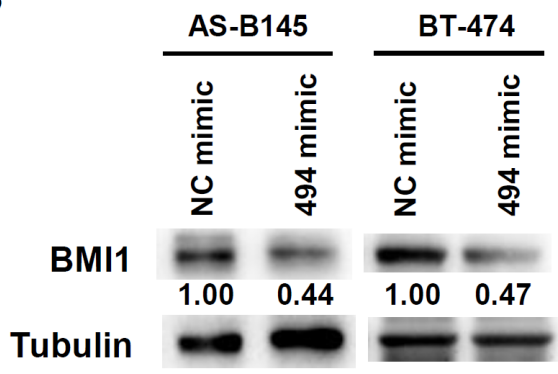

Figure 4: BMI1 is a target of miR-494-3p. (A) The alignment of BMI1 3'-UTR and miR-494-3p was obtained from the website of MICRORNA. ORG (http://www.microrna.org). 293-T or AS-B145 cells were transfected with a negative control (NC) mimic or miR-4943p (494-3p) mimic at a concentration of $100 \mathrm{nM}$ together with wildtype BMI1 3'-UTR (BMI1 3'-UTR WT) or mutant from (BMI1 3'UTR del 762-768) for $48 \mathrm{~h}$ and determined luciferase activities. Data were presented as mean \pm SD. ${ }^{*}, P<0.05 ;{ }^{* *}, P<0.01$. (B) AS-B145 or BT-474 cells were firstly cultured into primary mammospheres, dissociated into single cell suspension and transfected with NC or 494-3p mimic at a concentration of $100 \mathrm{nM}$ for 48 hours. BMI1 expression was then determined by western blot. Inset values indicate protein expression normalized to tubulin. The experiments were repeated at least two times and data from one experiment were presented. 
A

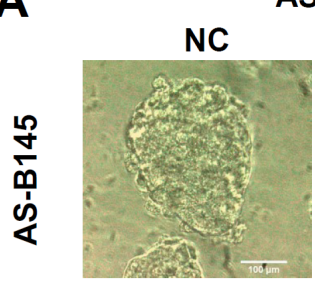

Primary

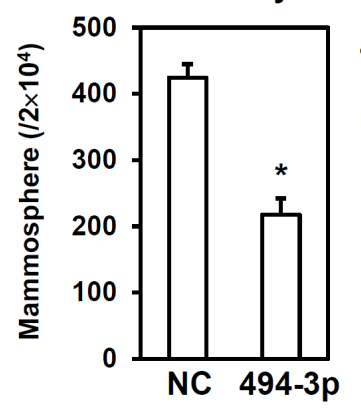

C

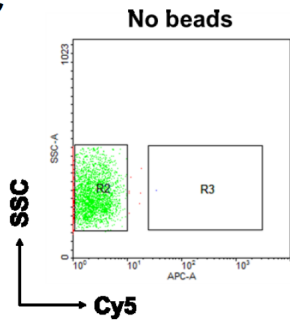

\section{Bmi1}

Tubulin

E

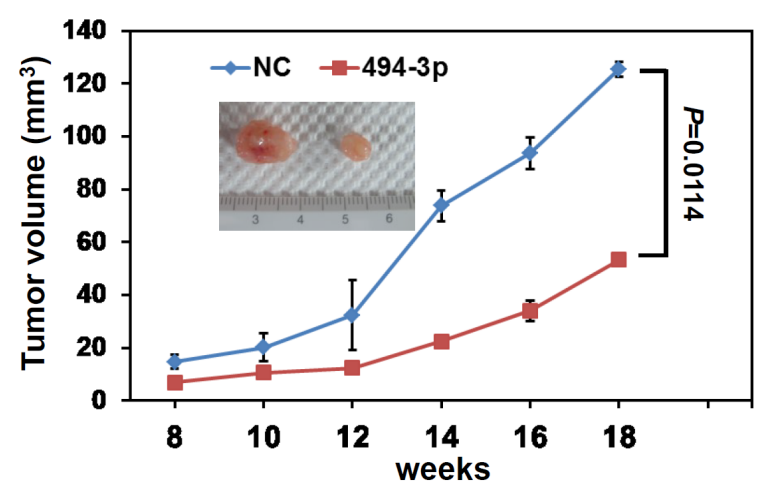

B

494-3p

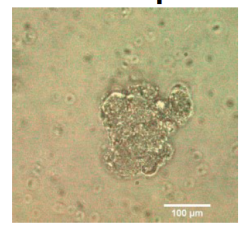

Secondary

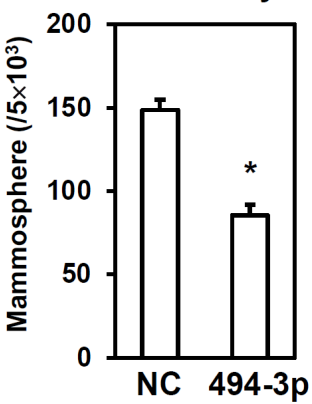

miR-494-3p SF-Cy5
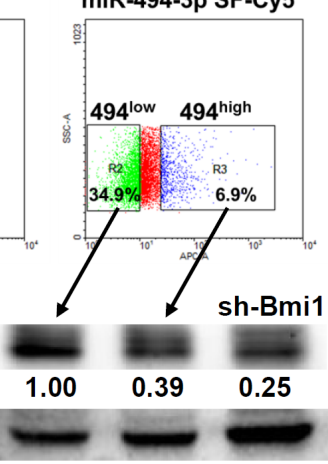$$
\text { E }
$$ 
A

GSE37405

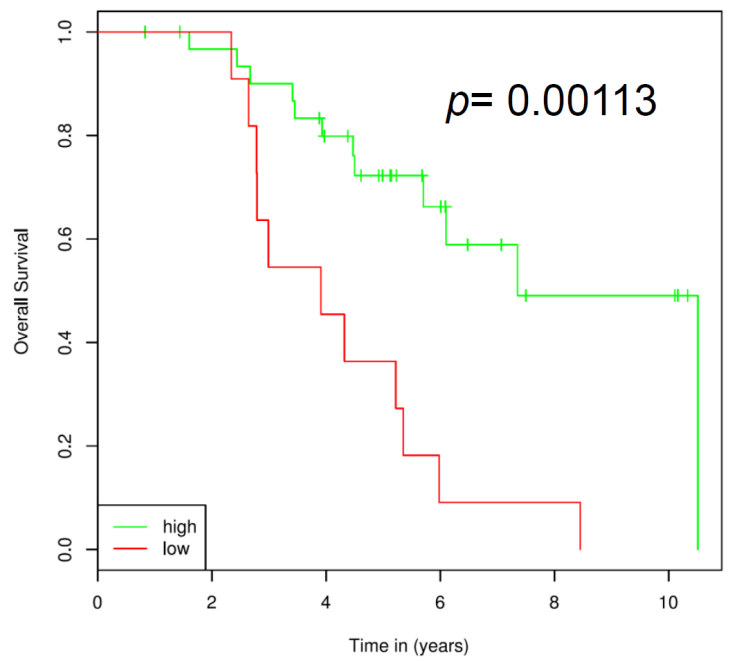

B

TCGA

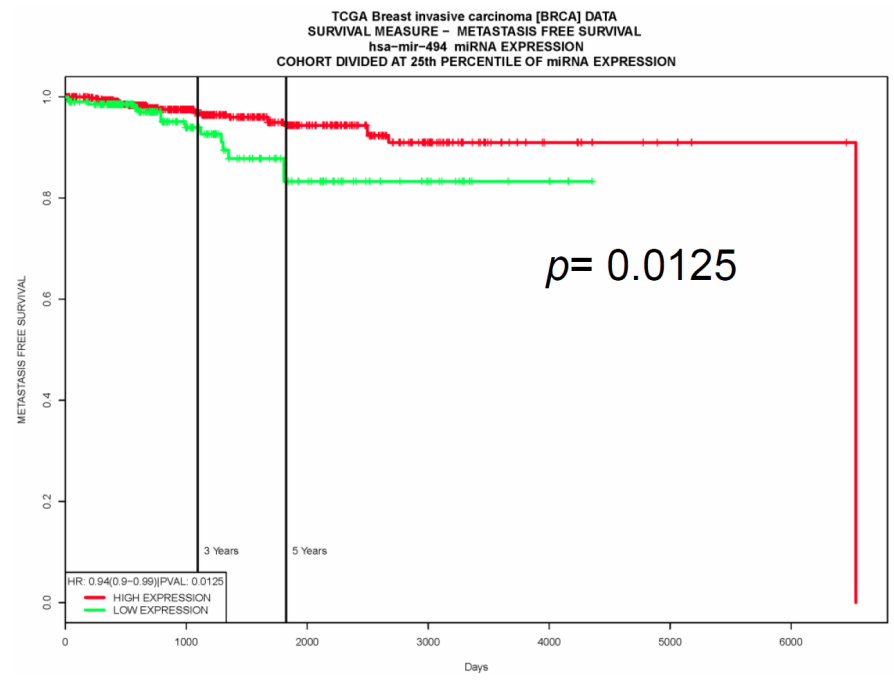

Figure 6: The expression of miR-494 is negatively correlated with survival time of breast cancer patients. The correlation between miR-494 expression and overall survival in a GSE37405 dataset of ER+ breast cancer patients (A) or metastasis free survival in TCGA invasive breast cancer dataset (B) was analyzed by MIRUMIR or PROGmiR V2 website.

A

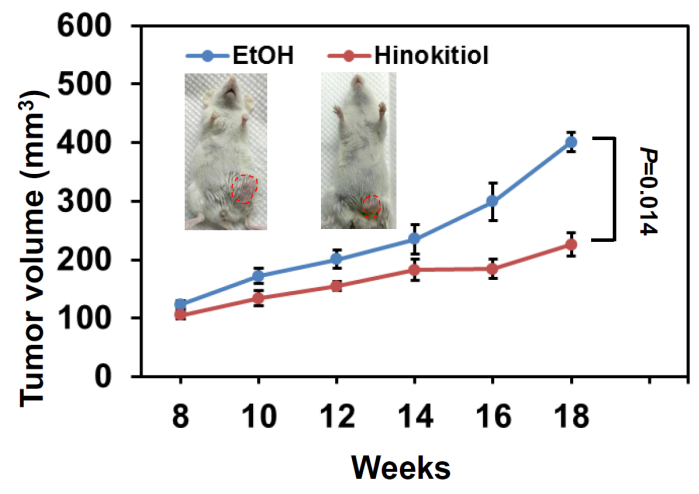

D

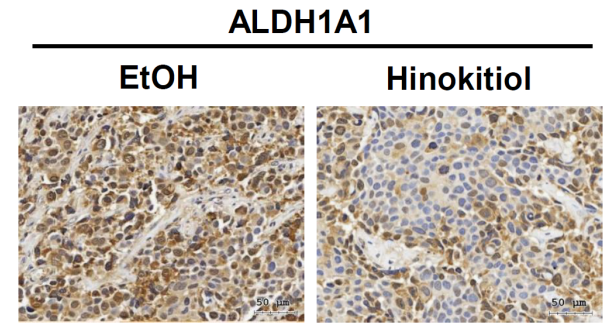

B

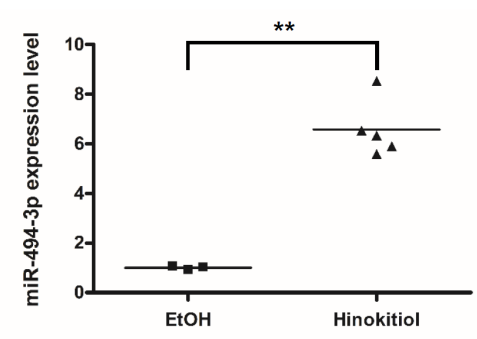

C

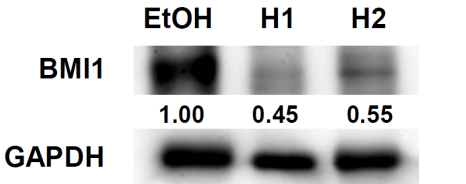

BMI1

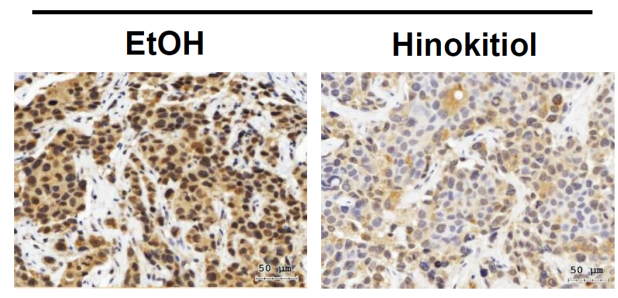

Figure 7: Hinokitiol reduces in vivo tumorigenicity of BCSCs. BT-474 cells were firstly cultured into mammospheres and $1 \times 10^{5}$ cells were injected into mammary fat pads of NOD/SCID mice for tumor growth. (A) The treatment of hinokitiol at a dose of $40 \mathrm{mg} / \mathrm{kg}$ was performed when tumors reached $100 \mathrm{~mm}^{3}$ by twice/week until 18 weeks. (B) miR-494-3p expression in each formed tumor was determined by qRT-PCR. ${ }^{* *}, P<0.01$. (C) BMI1 expression in tumors was determined by western blot. $\mathrm{H} 1$ or $\mathrm{H} 2$ represented independent tumor samples from hinokitiol treated mice. GAPDH was used as protein loading control. The inserted numbers indicated the relative expression level of BMI1 when compared to the EtOH treated sample. (D) The expression of ALDH1A1 and BMI1 in formed tumors was determined by immunohistochemistry. 
miR-494-3p oligos as an enhancer is also recommended to facilitate the therapeutic efficiency of hinokitiol in the future. In conclusion, these data demonstrated that hinokitiol could suppress the self-renewal and tumorigenicity of BCSCs through miR-494-3p-mediated BMI1 inhibition. These findings suggest that hinokitiol has a potential to be developed as a chemoprevention agent in breast cancer.

\section{MATERIALS AND METHODS}

\section{Cell lines and reagents}

AS-B145 cells were cultured in Minimum Essential Medium (MEM) Alpha Medium supplemented with $2 \mathrm{mM}$ L-glutamine, $10 \%$ heat-inactivated fetal bovine serum and $5 \mu \mathrm{g} / \mathrm{ml}$ insulin at $37^{\circ} \mathrm{C}$ in $5 \% \mathrm{CO}_{2}$. BT-474 cells were cultured in Dulbecco's modified Eagle medium and Ham's F-12 (DMEM/F12) medium (1:1) supplemented with 2 $\mathrm{mM}$ L-glutamine and $10 \%$ heat-inactivated fetal bovine serum (FBS) at $37^{\circ} \mathrm{C}$ in $5 \% \mathrm{CO}_{2}$. Hinokitiol was purchased from Sigma-Aldrich (Sigma-Aldrich, St. Louis, MO) and dissolved in absolute ethanol as a stock of $100 \mathrm{mM}$.

\section{Cell viability assay}

Cells were treated with a sequential concentration of hinokitiol $(0-1000 \mu \mathrm{M})$ and cultured for $48 \mathrm{~h}$. Cell survival was assessed using WST-1 reagent by measuring absorbance at 440nm wavelength. The half maximal inhibitory concentration (IC50) value was calculated by GraFit software (version 7, Erithacus Software Ltd., Surrey, UK)

\section{Immunoblot analysis}

The total protein concentration in each sample was determined with the bicinchoninic acid (BCA) protein assay (Pierce Biotechnology, Rockford, IL, USA). Proteins were separated by SDS-PAGE, transferred onto Polyvinylidene Fluoride (PVDF) membranes (Pall Corporation, Port Washington, NY, USA), and probed with rabbit polycolnal anti-BMI1 (Novus Biologicals, Littleton, $\mathrm{CO}$, USA) or rabbit polyclonal anti-GAPDH (GeneTex International Corporation, Hsinchu City, Taiwan). Horseradish peroxidase-conjugated goat anti-rabbit IgG polyclonal antibody (GeneTex) was used as the secondary antibody followed by incubation with T-Pro LumiFast Chemiluminescent Substrate (JF Ji-Feng Biotechnology, New Taipei City, Taiwan) and the signals were visualized and captured with a FUSION Solo S Imaging system (Vilber Lourmat, MArne-la-Valée, France). The signals were quantified with ImageJ software (version 1.51i, National Institute of Mental Health, Bethesda, MA, USA). For quantification of BMI1 protein in xenograft tumors by western bot, $10 \mu \mathrm{m}$ of paraffin embedded tumors were sliced and proteins were extracted according to the protocol of Guo et al. [40].

\section{Mammosphere cultivation}

Mammopshere cultivation was performed as previously described [41, 42]. Briefly, Cells were suspended into DMEM/F12 medium supplemented with 0.4\% bovine serum albumin (Sigma-Aldrich), $10 \mathrm{ng} /$ $\mathrm{ml}$ epidermal growth factor (PeproTech Asia, Rehovot, Israel), $10 \mathrm{ng} / \mathrm{ml}$ basic fibroblast growth factor (Sino Biological Inc., Beijing, China), 0.5X B27 supplement (Thermo Fisher Scientific, Waltham, MA, USA), $5 \mu \mathrm{g} / \mathrm{ml}$ insulin (Sigma-Aldrich), $1 \mu \mathrm{g} / \mathrm{ml}$ hydrocortisone (SigmaAldrich) and $4 \mu \mathrm{g} / \mathrm{ml}$ heparin (Sigma-Aldrich) and seeded into ultralow attachment 6-well-plate (Greiner Bio-One $\mathrm{GmbH}$, Kremsmünster, Austria) at a density of $1 \times 10^{4} / \mathrm{ml}$. The number of formed primary mammospheres with a diameter larger than $50 \mu \mathrm{m}$ was counted under an inverted microscopy. Primary mammospheres were harvested by filtering with $70 \mu \mathrm{m}$ cell strainer (BD Biosciences), dissociated into single cell suspension by HyQTase and performed secondary mammosphere formation as the protocol of primary mammosphere cultivation.

\section{Quantitative reverse transcription-polymerase chain reaction (qRT-PCR)}

qRT-PCR was performed as previously described [27]. Briefly, total RNA was extracted and purified by Quick-RNA ${ }^{\mathrm{TM}}$ MiniPrep Plus (Zymo Research Corp, Irvine, CA, USA). $1 \mu \mathrm{g}$ total RNA was used for complementary DNA (cDNA) conversion by RevertAid First Strand cDNA Synthesis Kit (Thermo Fisher Scientific). For miR-494-3p detection, a specific RT primer for miR-494-3p (Guangzhou RiboBio Co., Ltd., Guangzhou, China) was used for cDNA conversion and qPCR was then performed by KAPA SYBR ${ }^{\circledR}$ FAST qPCR Kit (Kapa Biosystems, Inc., Wilmington, MA, USA) and StepOnePlus ${ }^{\mathrm{TM}}$ Real-Time PCR System (Thermo Fisher Scientific) with specific qPCR primer pair (Guangzhou RiboBio Co., Ltd.) under a condition as described in [27]. RNU6B was used as internal control for analyzing miR494-3p expression. The primers sequences for detection of BMII and MRPL19 were used as described in [27].

\section{Transfection}

The transfection of plasmid DNA, miRNA mimic or inhibitor was performed by TurboFect Transfection Reagent (Thermo Fisher Scientific) with the manufacture's protocol. Briefly, $100 \mathrm{nM}$ miRNA mimic or inhibitor (purchased from Guangzhou RiboBio Co., Ltd.) or $1 \mu \mathrm{g}$ of pCMV14-3X flag or pCMV-BMI1-flag was complexed with transfection reagent as a ratio of $1 \mu \mathrm{g}$ nuclei acid: 2 $\mu l$ reagent at room temperature for 15 minutes and then 
added into wells of 6-well-plates with cell attachment at $60 \%$ confluency. Cells were then harvested at $48 \mathrm{~h}$ post transfection for further experiments.

\section{Constructs and luciferase-based reporter assay}

Human BMI1 gene was amplified by PCR from cDNA of BT-474 cells and cloned into pCMV14-3X flag vector with following primers: KpnI-BMI1-F (5'CgCggTAccATgCATCgAACAACgAgAATC-3') and BMI1ns-BamHI-R (5'-AgCggATCCACCAgAAgAAgTT gCTgATgAC-3'). The firefly luciferase reporter plasmid with full length BMI1 3'-UTR was purchased from OriGene Technologies, Inc. (Rockville, MD, USA) and a reporter plasmid of mutant BMI1 3'-UTR with a deletion of putative miR-494-3p binding region (deletion of nucleotide positions from 762 to 768 in 3'-UTR of BMI1) was constructed with site-directed mutagenesis kit as described in [27]. For reporter assay, the firefly luciferase reporter plasmid was mixed with a renilla luciferase plasmid as a ratio of 50:1 and transfected together with miR-494-3p mimic or negative control mimic into $293 \mathrm{~T}$ or BT-474 cells. The luciferase activity was detected at $48 \mathrm{~h}$ post transfection as described in [27].

\section{Fluorescence-activated cell sorting (FACS)}

ALDH+ BCSCs within BT-474 mammospheres were detected by ALDEFLUOR assay (StemCell Technologies, Inc., Vancouver, BC, Canada) according to our previous report [43]. The fluorescence signals were analyzed by COULTER ${ }^{\mathrm{TM}}$ Epics XL flow cytometry (Beckman Coulter, Inc. Brea, CA, USA). In order to sort the differential miR-494-3p expressing cells, the Smartflare beads was used (Merck Millipore, Temecula, CA, USA). BT-474 cells were grew in a $10 \mathrm{~cm}$ dish for $80 \%$ confluency with $8 \mathrm{ml}$ culture medium, added $8 \mu \mathrm{l}$ miR-494-3p Smartflare-Cy5 beads and then incubated at $37^{\circ} \mathrm{C}$ for 16 hours. After incubation, cells were harvested with trypsin/EDTA and suspended in DMEM/F12 supplemented with 5\% FBS and performed cell sorting with FACSAria cell sorter (BD Biosciences).

\section{Human breast cancer xenograftment model}

All the animal studies were operated following a protocol approved by Institutional Animal Care \& Utilization Committee of Chung Shan Medical University. For analysis of the expression of miR-494-3p to the tumorigenicity of BT-474 cells, cells were transduced with lentivirus carrying negative control or miR-494$3 \mathrm{p}$ precursor which were purchased from BioSettia (San Diego, CA, USA) for 3 days and injected into mammary fads of NOD/SCID mice (purchased from National Laboratory Animal Center, Taipei, Taiwan) as $1 \times 10^{6}$ cells $/ 50 \mu \mathrm{l} \mathrm{Matrigel} /$ site. For examine the in vivo therapeutic effect of hinokitiol, BT-474 cells were firstly cultured into secondary mammospheres and dissociated by HyQTase treatment. The dissociated secondary mammosphere cells were suspended in $2.5 \mathrm{mg} / \mathrm{ml}$ Matrigel (BD Biosciences) and injected into mammary fat pads as $2 \times 10^{4}$ cells $/ 50 \mu \mathrm{l} /$ site. Hinokitiol treatment was performed intraperitoneally when tumors reached $100 \mathrm{~mm}^{3}$. Tumor volume was calculated as $\mathrm{d}^{2} \times \mathrm{D} \times \pi / 6$ where $\mathrm{d}$ and $\mathrm{D}$ were the shortest and longest diameter in $\mathrm{mm}$, respectively [44].

\section{Immunohistochemistric analysis}

Xenografted tumors were harvested, fixed with 3.7\% formaldehyde and embedded into paraffin. $5 \mu \mathrm{m}$ sections were sliced and the expression of BMI1 or ALDH1A1 was detected by polyclonal rabbit anti-BMI1 antibody (Novus Biologicals, LLC) or polyclonal rabbit anti-ALDH1A1 antibody (GeneTex Inc.) followed by a standard avidinbiotin-peroxidase complex method. 3,3'-Diaminobenzidine (DAKO, Carpinteria, CA) was then used to detect the antibody binding. The images of sections were scanned by TissueFAXS Plus (TissueGnostics GmbH, Vienna, Austria).

\section{Analysis of the association between miR-494-3p expression and overall survival rate in breast cancer patients}

The association between miR-494-3p expression and overall survival rate of breast cancer patients was analyzed using public breast cancer datasets of GSE37405 and TCGA by online analysis tools. The dataset of GSE37405 was analyzed by MIRUMIR website (http:// www.chemoprofiling.org/cgi-bin/GEO/MIRUMIR/ web_run_MIRUMIR.V1.pl) whereas TCGA dataset was analyzed by PROGmiR V2 website (http://xvm145. jefferson.edu/progmir/).

\section{Statistical analysis}

One-way analysis of variance (one-way ANOVA) was used to identify differences between experimental groups and the control group. A $P$ value less than 0.05 was considered to be statistically significant.

\section{CONFLICTS OF INTEREST}

The authors declare no conflicts of interest.

\section{GRANT SUPPORT}

This work is supported by Ministry of Science and Technology in Taiwan (grant No. MOST 103-2314-B-040015-MY3). 


\section{REFERENCES}

1. Hermann PC, Bhaskar S, Cioffi M, Heeschen C. Cancer stem cells in solid tumors. Semin Cancer Biol. 2010; 20: 77-84.

2. Dalerba P, Cho RW, Clarke MF. Cancer stem cells: models and concepts. Annu Rev Med. 2007; 58: 267-284.

3. Reya T, Morrison SJ, Clarke MF, Weissman IL. Stem cells, cancer, and cancer stem cells. Nature. 2001; 414: 105-111.

4. Ajani JA, Song S, Hochster HS, Steinberg IB. Cancer Stem Cells: The Promise and the Potential. Seminars in oncology. 2015; 42: S3-S17.

5. Bouvard C, Barefield C, Zhu S. Cancer stem cells as a target population for drug discovery. Future Med Chem. 2014; 6: 1567-1585.

6. Al-Hajj M, Wicha MS, Benito-Hernandez A, Morrison SJ, Clarke MF. Prospective identification of tumorigenic breast cancer cells. Proc Natl Acad Sci U S A. 2003; 100: 3983-3988.

7. Ginestier C, Hur MH, Charafe-Jauffret E, Monville F, Dutcher J, Brown M, Jacquemier J, Viens P, Kleer CG, Liu S, Schott A, Hayes D, Birnbaum D, et al. ALDH1 is a marker of normal and malignant human mammary stem cells and a predictor of poor clinical outcome. Cell stem cell. 2007; 1: 555-567.

8. Lee CH, Yu CC, Wang BY, Chang WW. Tumorsphere as an effective in vitro platform for screening anti-cancer stem cell drugs. Oncotarget. 2016; 7: 1215-1226. doi: 10.18632/ oncotarget.6261.

9. Weiswald LB, Bellet D, Dangles-Marie V. Spherical cancer models in tumor biology. Neoplasia. 2015; 17: 1-15.

10. Vlashi E, Kim K, Lagadec C, Donna LD, McDonald JT, Eghbali M, Sayre JW, Stefani E, McBride W, Pajonk F. In vivo imaging, tracking, and targeting of cancer stem cells. Journal of the National Cancer Institute. 2009; 101: 350-359.

11. Liu S, Dontu G, Mantle ID, Patel S, Ahn NS, Jackson KW, Suri P, Wicha MS. Hedgehog signaling and Bmi-1 regulate self-renewal of normal and malignant human mammary stem cells. Cancer research. 2006; 66: 6063-6071.

12. Datta S, Hoenerhoff MJ, Bommi P, Sainger R, Guo WJ, Dimri M, Band H, Band V, Green JE, Dimri GP. Bmi-1 cooperates with H-Ras to transform human mammary epithelial cells via dysregulation of multiple growth-regulatory pathways. Cancer research. 2007; 67: 10286-10295.

13. Dimri M, Kang M, Dimri GP. A miR-200c/141-BMI1 autoregulatory loop regulates oncogenic activity of BMI1 in cancer cells. Oncotarget. 2016; 7: 36220-36234. doi: 10.18632/oncotarget.8811.

14. Bartel DP. MicroRNAs: genomics, biogenesis, mechanism, and function. Cell. 2004; 116:281-297.

15. Faversani A, Amatori S, Augello C, Colombo F, Porretti L, Fanelli M, Ferrero S, Palleschi A, Pelicci PG, Belloni E, Ercoli G, Degrassi A, Baccarin M, et al. miR-494-3p is a novel tumor driver of lung carcinogenesis. Oncotarget. 2017; 8: 7231-7247. doi: 10.18632/oncotarget.13933.
16. Lim L, Balakrishnan A, Huskey N, Jones KD, Jodari M, Ng R, Song G, Riordan J, Anderton B, Cheung ST, Willenbring H, Dupuy A, Chen X, et al. MicroRNA-494 within an oncogenic microRNA megacluster regulates G1/S transition in liver tumorigenesis through suppression of mutated in colorectal cancer. Hepatology. 2014; 59: 202-215.

17. Kim WK, Park M, Kim YK, Tae YK, Yang HK, Lee JM, Kim H. MicroRNA-494 downregulates KIT and inhibits gastrointestinal stromal tumor cell proliferation. Clinical cancer research. 2011; 17: 7584-7594.

18. Shen PF, Chen XQ, Liao YC, Chen N, Zhou Q, Wei Q, Li X, Wang J, Zeng H. MicroRNA-494-3p targets CXCR4 to suppress the proliferation, invasion, and migration of prostate cancer. Prostate. 2014; 74: 756-767.

19. Zhan MN, Yu XT, Tang J, Zhou CX, Wang CL, Yin QQ, Gong XF, He M, He JR, Chen GQ, Zhao Q. MicroRNA-494 inhibits breast cancer progression by directly targeting PAK1. Cell Death Dis. 2017; 8: e2529.

20. Shih YH, Lin DJ, Chang KW, Hsia SM, Ko SY, Lee SY, Hsue SS, Wang TH, Chen YL, Shieh TM. Evaluation physical characteristics and comparison antimicrobial and anti-inflammation potentials of dental root canal sealers containing hinokitiol in vitro. PloS one. 2014; 9: e94941.

21. Morita Y, Sakagami Y, Okabe T, Ohe T, Inamori Y, Ishida $\mathrm{N}$. The mechanism of the bactericidal activity of hinokitiol. Biocontrol Sci. 2007; 12: 101-110.

22. Lee YS, Choi KM, Kim W, Jeon YS, Lee YM, Hong JT, Yun YP, Yoo HS. Hinokitiol inhibits cell growth through induction of S-phase arrest and apoptosis in human colon cancer cells and suppresses tumor growth in a mouse xenograft experiment. J Nat Prod. 2013; 76: 2195-2202.

23. Liu S, Yamauchi H. p27-Associated G1 arrest induced by hinokitiol in human malignant melanoma cells is mediated via down-regulation of $\mathrm{pRb}, \mathrm{Skp} 2$ ubiquitin ligase, and impairment of Cdk2 function. Cancer letters. 2009; 286: 240-249.

24. Ido $\mathrm{Y}$, Muto N, Inada A, Kohroki J, Mano M, Odani T, Itoh N, Yamamoto K, Tanaka K. Induction of apoptosis by hinokitiol, a potent iron chelator, in teratocarcinoma F9 cells is mediated through the activation of caspase-3. Cell Prolif. 1999; 32: 63-73.

25. Wang WK, Lin ST, Chang WW, Liu LW, Li TY, Kuo CY, Hsieh JL, Lee CH. Hinokitiol induces autophagy in murine breast and colorectal cancer cells. Environ Toxicol. 2014.

26. Dontu G, Abdallah WM, Foley JM, Jackson KW, Clarke MF, Kawamura MJ, Wicha MS. In vitro propagation and transcriptional profiling of human mammary stem/ progenitor cells. Genes Dev. 2003; 17: 1253-1270.

27. Weng JH, Yu CC, Lee YC, Lin CW, Chang WW, Kuo YL. miR-494-3p Induces Cellular Senescence and Enhances Radiosensitivity in Human Oral Squamous Carcinoma Cells. Int J Mol Sci. 2016; 17.

28. Amatya VJ, Mawas AS, Kushitani K, Mohi El-Din MM, Takeshima Y. Differential microRNA expression profiling of mesothelioma and expression analysis of miR-1 
and miR-214 in mesothelioma. International journal of oncology. 2016; 48: 1599-1607.

29. Li LH, Wu P, Lee JY, Li PR, Hsieh WY, Ho CC, Ho CL, Chen WJ, Wang CC, Yen MY, Yang SM, Chen HW. Hinokitiol induces DNA damage and autophagy followed by cell cycle arrest and senescence in gefitinib-resistant lung adenocarcinoma cells. PloS one. 2014; 9: e104203.

30. Hibino S, Saito Y, Muramatsu T, Otani A, Kasai Y, Kimura $\mathrm{M}$, Saito H. Inhibitors of enhancer of zeste homolog 2 (EZH2) activate tumor-suppressor microRNAs in human cancer cells. Oncogenesis. 2014; 3: e104.

31. Alzrigat M, Parraga AA, Agarwal P, Zureigat H, Osterborg A, Nahi H, Ma A, Jin J, Nilsson K, Oberg F, Kalushkova A, Jernberg-Wiklund H. EZH2 inhibition in multiple myeloma downregulates myeloma associated oncogenes and upregulates microRNAs with potential tumor suppressor functions. Oncotarget. 2017; 8: 10213-10224. doi: 10.18632/oncotarget.14378.

32. Tu DG, Yu Y, Lee CH, Kuo YL, Lu YC, Tu CW, Chang WW. Hinokitiol inhibits vasculogenic mimicry activity of breast cancer stem/progenitor cells through proteasomemediated degradation of epidermal growth factor receptor. Oncol Lett. 2016; 11: 2934-2940.

33. Du WW, Fang L, Yang X, Sheng W, Yang BL, Seth A, Zhang Y, Yang BB, Yee AJ. The role of versican in modulating breast cancer cell self-renewal. Mol Cancer Res. 2013; 11: 443-455.

34. Yang J, Liao D, Chen C, Liu Y, Chuang TH, Xiang R, Markowitz D, Reisfeld RA, Luo Y. Tumor-associated macrophages regulate murine breast cancer stem cells through a novel paracrine EGFR/Stat3/Sox-2 signaling pathway. Stem cells. 2013; 31: 248-258.

35. Chen HH, Huang WT, Yang LW, Lin CW. The PTENAKT-mTOR/RICTOR Pathway in Nasal Natural Killer Cell Lymphoma Is Activated by miR-494-3p via PTEN But Inhibited by miR-142-3p via RICTOR. The American journal of pathology. 2015; 185: 1487-1499.

36. Li J, Wang L, Liu Z, Zu C, Xing F, Yang P, Yang Y, Dang X, Wang K. MicroRNA-494 inhibits cell proliferation and invasion of chondrosarcoma cells in vivo and in vitro by directly targeting SOX9. Oncotarget. 2015; 6: 26216-26229. doi: 10.18632/oncotarget.4460.

37. Costa-Pinheiro P, Ramalho-Carvalho J, Vieira FQ, TorresFerreira J, Oliveira J, Goncalves CS, Costa BM, Henrique $\mathrm{R}$, Jeronimo C. MicroRNA-375 plays a dual role in prostate carcinogenesis. Clin Epigenetics. 2015; 7: 42.

38. Szafranska AE, Davison TS, John J, Cannon T, Sipos B, Maghnouj A, Labourier E, Hahn SA. MicroRNA expression alterations are linked to tumorigenesis and non-neoplastic processes in pancreatic ductal adenocarcinoma. Oncogene. 2007; 26: 4442-4452.

39. Yuan W, Yuan Y, Zhang T, Wu S. Role of Bmi-1 in regulation of ionizing irradiation-induced epithelialmesenchymal transition and migration of breast cancer cells. PloS one. 2015; 10: e0118799.

40. Guo H, Liu W, Ju Z, Tamboli P, Jonasch E, Mills GB, Lu Y, Hennessy BT, Tsavachidou D. An efficient procedure for protein extraction from formalin-fixed, paraffin-embedded tissues for reverse phase protein arrays. Proteome Sci. 2012; 10: 56.

41. Lu KT, Wang BY, Chi WY, Chang-Chien J, Yang JJ, Lee HT, Tzeng YM, Chang WW. Ovatodiolide Inhibits Breast Cancer Stem/Progenitor Cells through SMURF2-Mediated Downregulation of Hsp27. Toxins (Basel). 2016; 8.

42. Chang WW, Lin RJ, Yu J, Chang WY, Fu CH, Lai AC, Yu JC, Yu AL. The expression and significance of insulin-like growth factor-1 receptor and its pathway on breast cancer stem/progenitors. Breast cancer research. 2013; 15: R39.

43. Wei L, Liu TT, Wang HH, Hong HM, Yu AL, Feng HP, Chang WW. Hsp27 participates in the maintenance of breast cancer stem cells through regulation of epithelialmesenchymal transition and nuclear factor-kappaB. Breast cancer research. 2011; 13: R101.

44. Weiss JM, Shivakumar R, Feller S, Li LH, Hanson A, Fogler WE, Fratantoni JC, Liu LN. Rapid, in vivo, evaluation of antiangiogenic and antineoplastic gene products by nonviral transfection of tumor cells. Cancer Gene Ther. 2004; 11: 346-353. 